\title{
Harmonisation des notations concernant la des- cription morphologique d'un pied de maïs (Zea mays L.)
}

Pliilippe GIRARDIN, Marie-Odile JORDAN, Didier PICARD \& Roland TRENDEL

I.N.R.A., Station d'Agronomie, Centre de Recherches de Colmar, F 68021 Colmar Cedex

RESUMÉ

Parallèlement aux 3 stades repères concernant les feuilles (ébauche foliaire, feuille " visible ", feuille " étalée "), il est suggéré de retenir les stades repères suivants pour les racines: ébauche racinaire, racine allongée, racine ramifiée.

Mots clés additionnels : Feuilles, racines, stades repères.

Additional key words : Leaves, roots, reference stages.

\section{INTRODUCTION}

Un premier essai de rationalisation du vocabulaire employé pour les descriptions morphologiques de plantules de maïs a été tenté par ONDERDONK \& KETCHESON (1972). Si les règles générales données par ces auteurs étaient suffisantes tant que l'on se limitait à l'étude des parties aériennes, il s'avère indispensable d'apporter quelques précisions complémentaires lorsqu'on aborde l'étude des racines. De plus, il apparaît utile de préciser, en langue française, les termes concernant les descriptions morphologiques relatives au maïs.

\section{STRUCTURE D'UN PIED DE MAÏS}

KiesSelbaCH (1949) a mis en évidence, le premier, l'existence, chez le maïs, d'unités élémentaires superposées : les phytomères. Chaque phytomère comprend (fig. 1) :

- un entrenœud surmonté d'un nœud,

- et éventuellement :

- une feuille (gaine, ligule et limbe) ou un homologue de feuille issu du nœud,

- un bourgeon axillaire qui avorte ou évolue

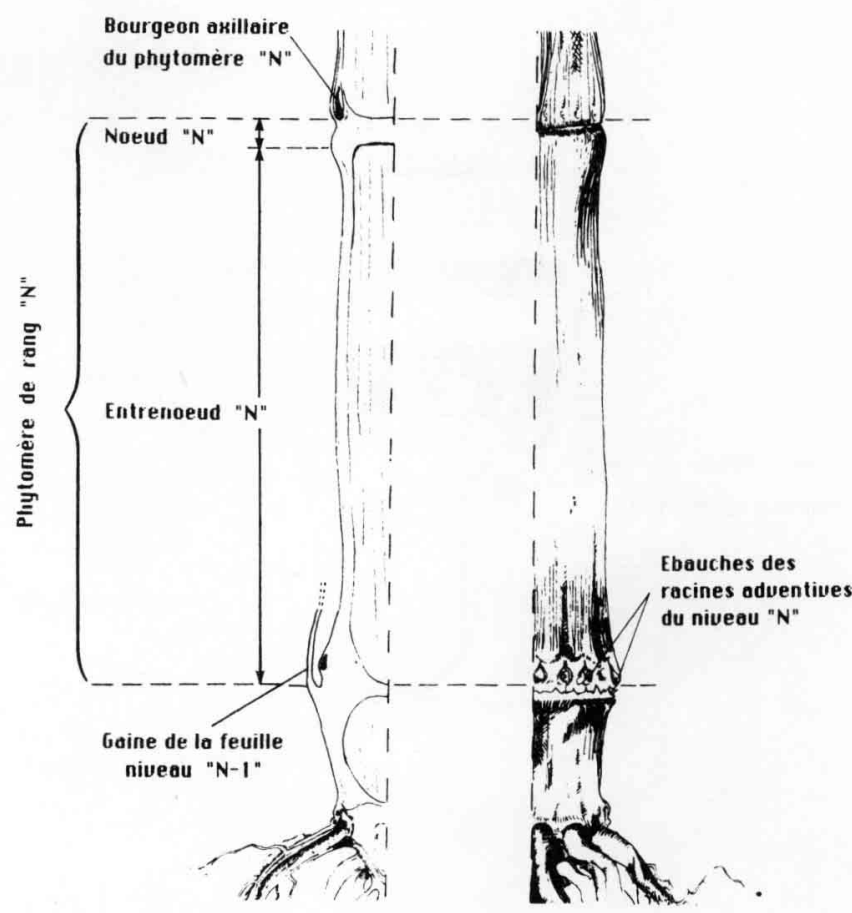

Figure 1

Détail d'un phytomère.

"Phytomere " detail. 
soit en talle soit en épi suivant les conditions de milieu,

- une série d'ébauches situées à la base de l'entrenœud qui peuvent donner naissance à des racines adventives.

A partir de ce schéma de base et compte tenu des propositions faites par ONDERDONG \& KETCHESON (1972), établies à partir de la terminologie relevée dans la bibliographie, et de l'étude de PICARD et al. (1985), il est possible de décrire un pied de maïs de la manière suivante (fig. 2) :

1) la $1^{\text {re }}$ racine à apparaître, en perçant la coléorhize, est la « racine séminale » qui peut porter des racines secondaires et tertiaires.

Par souci d'homogénéité, on pourrait individualiser un phytomère $\mathrm{n}^{\circ} 0$, composé d'un entrenœud réduit (l'hypocotyle), d'un nœud (le noud scutellaire), d'un homologue de feuille (le scutellum) (KIESSELBACH, 1949 ; AVERY, 1930) et d'une racine (la racine séminale) ;

2) les racines qui apparaissent immédiatement après, au nombre de 0 à 8 , (SIEMENS, 1929) appartiennent au $1^{\text {er }}$ entrencud (E1) qui est appelé " mésocotyle ». Elles sont souvent comptabilisées parmi les séminales ou appelées séminales secondaires (SIEMENS, 1929) bien que ce soit, comme nous le verrons ultérieurement, les $1^{\text {res }}$ racines primaires adventives. Contrairement à ce qui se passe pour les entrenœuds suivants, les racines peuvent être émises tout le long du mésocotyle. La longueur de celui-ci varie suivant la profondeur de semis. Il est surmonté par le noud coléoptilaire qui donne naissance au coléoptile. Cet ensemble constitue le $1^{\text {er }}$ phytomère ;

3) la $1^{\text {re }}$ feuille vraie (F1) est celle qui naît au $3^{\mathrm{e}}$ nœud à la partie supérieure du $2^{\mathrm{e}}$ entrenœud (E2). Ce phytomère comporte de 3 à 6 racines (PICARD et $a l ., 1985$; JORDAN et al., en préparation). En consé-

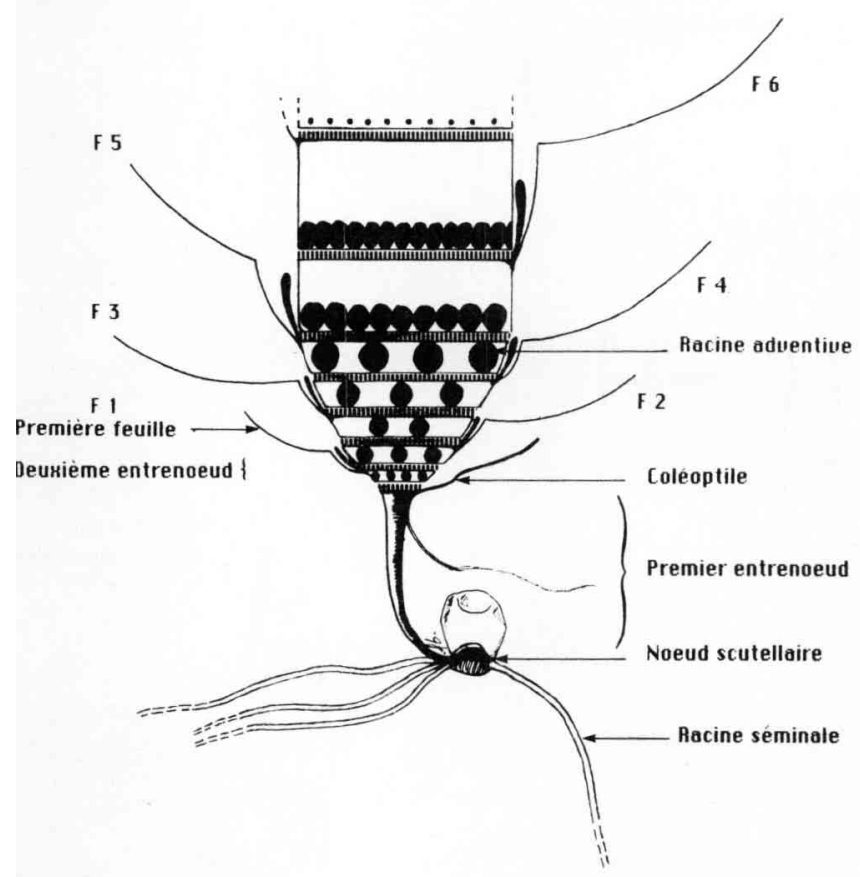

Figure 2

Schéma d'un plant de maïs.

Mais plant design. quence de quoi, la feuille vraie de rang $n$ (en comptant de bas en haut) appartient au phytomère de rang $n+1$. La gaine de cette feuille $(\mathrm{Fn})$ masque, puis est quelquefois transpercée par les racines de l'entrenœud $n+2$.

Le nombre de racines portées par le mésocotyle est fonction de la variété et des conditions de milieu (de 0 à 5 pour le cultivar Dea) (PICARD et al., 1985 ; SIEMENS, 1929) : ces racines sont d'autant plus nombreuses que la racine séminale se développe moins bien (MEssiaEn, 1963). La longueur des entrenœuds E2 et E5 est sensiblement égale au diamètre des racines primaires qui en sont issues ; il est donc parfois difficile de distinguer ces entrenœuds qui constituent la «base de la tige " de forme pyramidale. Au-delà de E5, pour la variété «Dea » (PICARD et al., 1985), les entrenœuds s'allongent et portent un nombre croissant d'ébauches racinaires. Le niveau du dernier entrenœud développant des racines allongées varie suivant les variétés (de E6 pour LG1 à E12 pour « Cuzco ») et les conditions de milieu (JORDAN et al., en préparation). Compte tenu de leurs caractéristiques anatomiques ou morphologiques, il n'y a pas de raison d'établir des distinctions entre les différents niveaux de racines ; il ne semble donc pas utile de retenir les termes racines d'ancrage ou racines aériennes.

\section{STADES REPÈRES POUR LES FEUILLES ET LES RACINES}

Il est convenu, en général, d'utiliser 3 stades repères pour les notations concernant les feuilles :

1. Initiation de la feuille au niveau du bourgeon terminal, ce stade sert à déterminer le plastochrone (ERICKSON \& MiCHELINI, 1957).

2. Stade "feuille visible ", l'extrémité apicale du limbe pointe hors du cornet foliaire. Ce stade est utilisé pour déterminer le phyllochrone (GOUNOT et al., 1980).

3. Stade "feuille étalée », la ligule est visible ; le limbe a atteint sa longueur maximum, mais la gaine peut encore s'allonger.

En ce qui concerne les racines primaires directement insérées sur la tige, il est possible de distinguer, quel que soit l'entrenœud considéré, 3 stades repères utilisables pour les notations phénologiques à l'œil nu (fig. 3) :

1. Stade « ébauche racinaire », le massif cellulaire donnant naissance à une racine est visible mais il n'a pas encore percé l'épiderme de la tige.

2. Stade « racine allongée », la racine a traversé l'épiderme de la tige, elle est fonctionnelle, mais elle ne possède pas encore de racines secondaires, elle peut être aérienne.

3. Stade "racine ramifiée », des ramifications d'ordre 2 (racines secondaires) se différencient sur les racines primaires et peuvent à leur tour porter des racines d'ordre 3 , voire d'ordre 4 .

L'ensemble des racines secondaires et d'ordres supérieurs issus d'une racine primaire, est appelé « réseau 


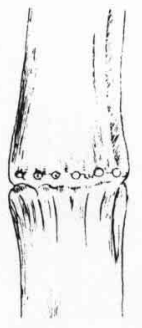

Stade I

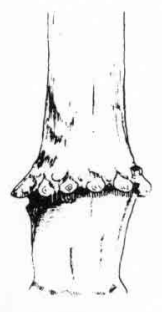

Stade 2

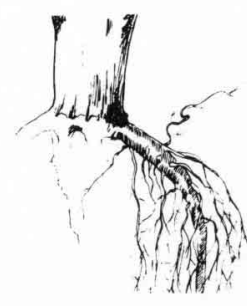

Stade 3 fhauches de racines

Bacines ollangees

Aacine ramiliee

Figure 3

Stades repères d'une racine primaire de maïs.

Reference stages of a maize primary root.

racinaire » (PICARD, 1976). L'ensemble des réseaux racinaires forment le «système racinaire ». Hormis la racine séminale, les autres racines sont toutes issues de la tige, ce sont des « racines adventives». Le terme couramment utilisé de "racines nodales" ne se justifie pas puisque les racines ne sont pas issues des

nœuds. Le terme «adventif » est à préférer à «coronaire » puisqu'il se peut qu'il n'y ait qu'une seule racine par phytomère, voire aucun, dans certains cas. En 3 semaines, l'ensemble des racines primaires appartenant à un même phytomère passe du stade ébauche au stade racine allongée, pour les $1^{\text {ers }}$ entrenœuds (MiLlet et al., en préparation). Cependant, il peut apparaître par la suite un certain nombre de racines supplémentaires de diamètre plus petit. Ainsi, on peut noter quelquefois, sur les entrenœuds supérieurs, l'apparition tardive de 2 racines de part et d'autre du bourgeon axillaire qui a avorté. Les autres racines de ce type appartiennent à un verticille situé au-dessus du verticille des $1^{\text {res }}$ racines apparues. Elles sont intercalées entre les précédentes, de diamètre plus faible et sont appelées "racines tardives".

L'emploi d'une notation unifiée pour les observations concernant le développement de l'ensemble d'un pied de maïs devrait faciliter les comparaisons entre études utilisant divers types de matériel végétal dans des conditions de milieux différents.

Reçu le 6 janvier 1986. Accepté le 30 juin 1986.

RÉFÉRENCES BIBLIOGRAPHIQUES

Avery G. S., 1930. Comparative anatomy and morphology of embryos and seedlings of maize, oat and wheat. Bot. Gaz., 19, 139.

Erickson R. O., Michelini F. J., 1957. The plastochron Index. Amer. J. Bot., 44, 297-305.

Gounot M., Atry M., N'Kandzas Uy O., 1980. Photosynthèse nette et rythme d'apparition de feuilles chez le dactyle (Dactylis glomerata L.): C. $R$. Acad. Sci., série D, 290, 1257-1260.

Kiesselbach T. A., 1949. The structure and reproduction of corn. Univ. of Nebraska Press, reed., 1980, 96 p.

Messiaen C. M., 1963. Physiologie du développement chez Zea mays. Ann. Epiphyt., 14, 1-90.
Onderdonk J. J., Ketcheson J. O., 1972. A standardization of terminology for the morphological description of corn seedling. Can. J. Plant Sci., 52, 1003-1006.

Picard D., 1976. Dynamique racinaire de Panicum maximum Jacq. et apport au sol de matière organique. Thèse Doc. es-Sci. Clermont Ferrand, 275 p.

Picard D., Jordan M. O., Trendel R., 1985. Rythme d'apparition des racines primaires du mais (Zea mays L.) : I. Etude détaillée pour une variété en un lieu donné. Agronomie, 5 (8), 667-676.

Siemens H. J., 1929. The development of secondary roots in corn seedlings. Sci. Agric., 9, 747-759. 